\title{
Assessment of Glaucoma with Ocular Thermal Images using GLCM Techniques
}

\author{
by N.Padmapriya ${ }^{1}$, N.Venkateswaran ${ }^{2}$, Toshitha Kannan ${ }^{3}$ and M. Sindhu Madhuri ${ }^{4}$ \\ 1,2,3,4 SSN College of Engineering, Chennai, Tamil Nadu, India, padmapriyan@ssn.edu.in
}

\begin{abstract}
This paper proposes a methodology for early detection and recognition of Glaucoma in ocular thermographs. Ocular thermography is an efficient tool not only to capture temperatures of corneal surface, but also to detect and visualize any changes on the Ocular surface temperature. The proposed method uses a linear transformation for preprocessing. Support vector machines (SVM) based classifier with the features collected from GLCM is used to classify the given ocular IR thermal image into Glaucoma from the normal eye. The efficacy of the proposed technique is proved over a number of ocular thermal image samples.
\end{abstract}

Keywords: Thermography, Glaucoma, Ocular Surface Disorder, Gray Level Co-occurrence Matrix (GLCM), Support vector machine (SVM).

\section{Introduction}

Infrared (IR) thermography is a non-contact and non-intrusive temperature measuring technique, capable of displaying real-time surface temperature distribution. Thermography is an investigative technique which allows rapid color- coded display of the temperature across a wide surface by means of infrared detection. Infrared thermography is used in satellite imaging, movement detection, security, surveillance, etc. In medicine, thermography has been extensively used in the diagnosis of breast cancer, diabetes neuropathy and peripheral vascular disorders. In addition, it has been used to detect problems associated with gynaecology, kidney transplantation, dermatology, heart, neonatal physiology, fever screening and brain imaging [1]. Infrared (IR) thermography provides information on the thermal, metabolic, and vascular conditions of the human body that may be used to interpret the pathophysiology information related to physical conditions. Studies and clinical observations have proven that IR thermography is a suitable indicator for distinguishing normal and abnormal physiology progresses, particularly for diseases in its early phase [1]. It is completely safe and uses no radiation.

In this paper, we are specifically interested in the mapping of ocular region and diagnosis of diseases pertaining to the same. Ocular thermography using infrared imaging systems has been used to investigate ocular tumors, retinoblastoma, glaucoma etc. The most common diseases that manifest in the eye are diabetic retinopathy, age-related macular degeneration, glaucoma and cardiovascular diseases. Above diseases damage the retina which can be observed in retinograms. In order to analyse the retinal structure and its changes over time, the retina should be imaged effectively. Retinal imaging techniques are developed in order to capture the retina morphology effectively and to model its changes over time. Fundus photography, Optical coherence tomography (OCT), Fluorescein angiography. Ocular IR thermography is a retinal imaging techniques that are widely used for the detection of various diseases and does not harm the subjects under investigation [2].

Glaucoma is invisible disease that can damage the eye's optic nerve and this leads to loss of vision and blindness. Eye has pressure just like our blood and an increase in intra ocular pressure (IOP) damages the optic nerve. Although the raised IOP is a significant risk factor for developing glaucoma, there is no set threshold for IOP that causes glaucoma. Glaucoma is similar to ocular hypertension, but with accompanying optic nerve damage [3]. To understand glaucoma it is important to consider aqueous humor, the clear watery fluid that circulates through the anterior chamber. This fluid is not related to tears, or to the dense jelly-like substance called vitreous humor contained in the rear chamber. The function of aqueous humor is to nourish the area around the iris and the cornea and it exerts pressure to maintain the shape of the eye [4].

Glaucoma can be broadly categorized as primary open angle glaucoma (POAG), closed angle glaucoma (CAG) or congenital. Open-angle glaucoma is the most common form of the disease. In open-angle glaucoma, even though the drainage angle is "open", the fluid passes too slowly through the meshwork drain. Since the fluid builds up, the pressure inside the eye rises to a level that may damage the optic nerve. When the optic nerve is damaged due to increased pressure, open-angle glaucoma and vision loss may result. Controlling pressure inside the eye is important. Most treatments for glaucoma are aimed at reducing IOP and its fluctuation. Treatment can be categorized as medical, laser and surgery [5].

In fundus image based glaucoma detection, cup to disc ratio (CDR) is a key indicator for the detection of glaucoma. Kavitha et. al. [3], proposed two methods to extract the disc automatically. The component analysis method 
and region of interest (ROI) based segmentation are used for the detection of disc. For the detection of the cup region, component analysis method is also used. Later the active contour method is used to plot the boundary accurately. Wiecek et. al. [6], presented new image processing tool for conversion of thermal and visual images, for application in medicine and biology. A novel method for area and distance evaluation based on statistical differencing is discussed. The aim is to compute thermal signatures for precisely defined areas of interest. The processing is based on local statistical characteristics of the image and found that they are useful for very non-homogenous images.

Duarte et. al. [7], provided improved characterization of thermal images, by developing a computational application. The limitations of existing software applications was overcome by designing an application that allows choosing any ROI, independently of its geometric shape and optimizing it for further processing. Morgan et. al.[8],discusses potential applications of ocular thermography. The paper discusses the dry eye syndrome and its diagnosis using thermal imaging in detail. Also, it examines the relationship between eye temperatures and ocular parameters. Bhattacharya and Deepa [9] proposed a two stage method to diagnose dry eye syndrome without direct eye contact. Thermal face image of the patient and Gray Level Co-occurrence Matrix is used for texture feature extraction. Tan et. al.[10], studied the performance of an automated algorithm which acquires corneal surface temperature for normal IR thermograms of different age groups. The automated algorithm suggested in this paper intelligently localizes cornea and removes eye lashes and reads temperature values without any human intervention. Tan et. al [1], reviewed the use and advantages of IR thermography in the field of ophthalmology. Different algorithms to acquire the ocular surface temperature (OST), which can be used for the diagnosis of ocular diseases, are discussed.

This paper is organized as follows: In section 2, we present the methodology that is followed in the classification of ocular thermal images for Glaucoma detection. Section 3 discusses the SVM based linear classification and presents the experimental results and section 4 concludes the paper.

\section{Methodology}

Figure 1 shows the flowchart of the proposed methodology for the detection of Glaucoma in ocular thermal images based on computing features from the Gray Level Co-occurrence Matrix (GLCM). Initially, the ocular thermal image is preprocessed by transforming the RGB image into YIQ format using a linear transformation or using Histogram Equalization. RGB is the most commonly used colour space. For processing of colour images the images can also be converted to other colour spaces such as HSI, YIQ, CIE-Lab and so on. This is followed by histogram equalization and noise filtering. GLCM is computed on the pre-processed image. Four features namely Energy, Homogeneity, Contrast and correlation are extracted using equations (1) to (4). The extracted features are used to train a Support vector machine (SVM) classifier.

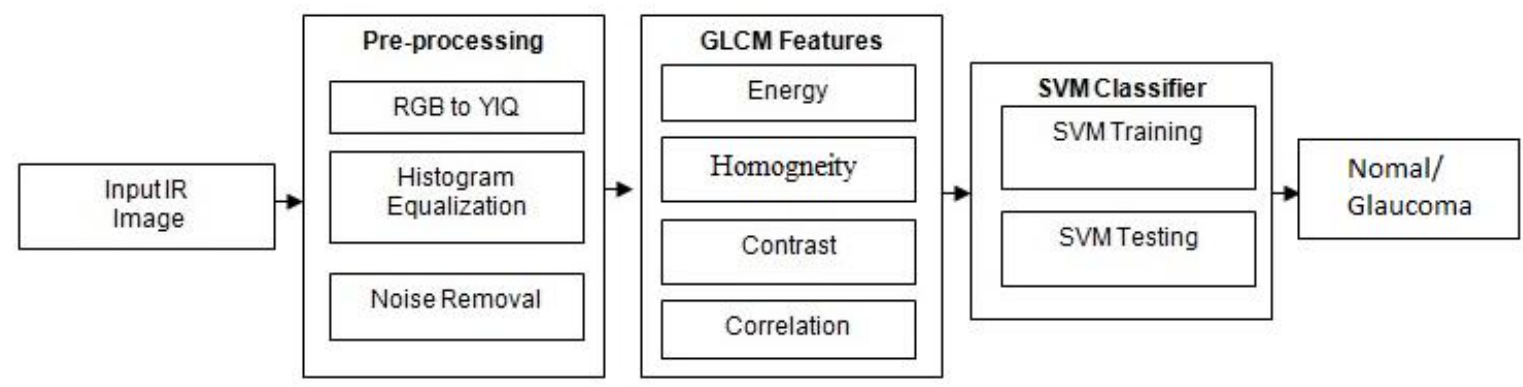

Fig. 1. Flowchart showing the Methodology followed

More advanced statistical information on thermal images can be derived from second order parameters. It is defined by a co-occurrence matrix [6]. Such a matrix represents the joint probability of two pixels having $i^{\text {th }}$ and $j^{\text {th }}$ intensity at the different distances $d$, in different directions. Co-occurrence matrix gives more information on intensity distribution over the whole image; it can effectively be used to separate and classify thermal images. The co-occurrence matrix is always square and diagonal with the dimension equal to the number of intensity levels in the image. After normalization we get the matrix of the probabilities $p(i, j)$. Normalization is done by dividing all elements by number of possible similar pixels for a given direction of analysis. There is a question of the size of the window for calculating the features. In practice, it can vary from $4 \times 4$ up to $32 \times 32$, but larger windows are statistically more significant [6]. Statistical features such as Energy, Local Homogeneity, Contrast and Correlation are extracted from GLCM in different directions. Contrast gives the variance of the elements of the GLCM. Energy provides the sum of squared elements in the GLCM, 
whereas, correlation measures the joint probability occurrence of the specified pixel pairs. Homogeneity measures the closeness of the distribution of elements in the GLCM. These parameters are defined by the following formulas.

$$
\begin{aligned}
\text { Energy } & =\sum_{i=0}^{L-1} \sum_{j=0}^{L-1} p^{2}(i, j) \\
\text { Local Homogeneity } & =\sum_{i=0}^{L-1} \sum_{j=0}^{L-1} \frac{p(i, j)}{1+|i-j|} \\
\text { Contrast } & =\frac{1}{(L-1)^{2}} \sum_{i=0}^{L-1} \sum_{j=0}^{L-1}(i-j)^{2} p(i, j) \\
\text { Correlation } & =\frac{\sum_{i=0}^{L-1} \sum_{j=0}^{L-1}(i \times j) p(i, j)-\mu_{h} \mu_{v}}{\sigma_{h} \sigma_{v}}
\end{aligned}
$$

In the above equations $\mu_{h}, \mu_{v}, \sigma_{h}$ and $\sigma_{v}$ are mean values and standard deviations for the elements of cooccurrence matrixes calculated for horizontal and vertical directions, respectively. $L$ represents the total number of grey levels used.

\section{Classification and Experiments}

\subsection{SVM Classifier}

In the previous section, we have outlined the method of extracting features from an ocular thermal image. An $M X M$ ocular IR thermal image is transformed into a 4 dimensional feature vector, whose parameters together preserve the information present in the image. The next step is to evaluate the discriminate power by means of a classifier. There are many classification techniques available in the literature. Support Vector Machine (SVM) based classification is adopted in this paper. SVM are supervised learning models with associated learning algorithms that analyse data and recognize patterns in an n-dimensional space. The function of the SVM classifier is to construct a hyper plane that separates the two different data sets with maximum margin between the data sets. In the case of support vector machine, an object is viewed as an n-dimensional vector and we want to separate such objects with an $n-1$ dimensional hyper plane. This is called a linear classifier.

Given a set of training examples for each class, the SVM machine learning algorithm builds a model for the given data set. The model now is used to assign any other example data into one class or the other, making it a nonprobabilistic binary linear classifier. The SVM approach seeks to find the optimal separating hyperplane between classes by focusing on the training classes that are placed at the edge of the class descriptors. These training classes are called as support vectors and they lie closest to the decision margin [11]. Only the support vectors determine the position of the hyperplane. The margin of the linear classifier is defined as the width that the boundary could be increased by before hitting the data point.

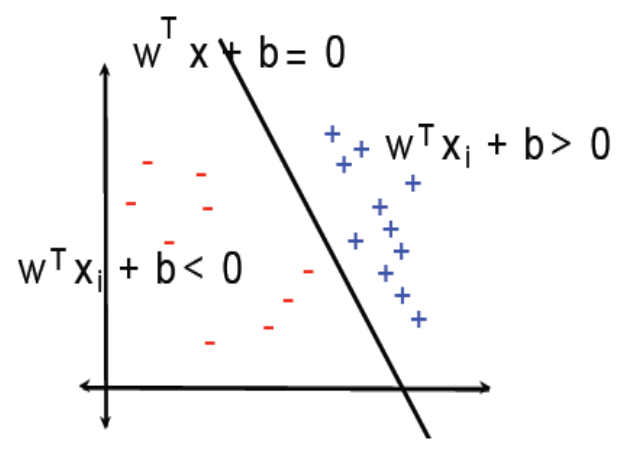

Fig. 2. SVM - classifier in 2-D space with a line separation 
Given training data $\left(x_{i}, y_{i}\right)$ for $i=1,2, \ldots \ldots . . N$, with $\left(x_{i} \in R^{d}\right)$ and $y_{i} \in\{+1,-1\}$, SVM learns a classifier $\left.f(x)\right)$

such that,

$$
f\left(x_{i}\right)=\left\{\begin{array}{c}
\geq 0, \text { then } \quad y_{i}=+1 \\
<0, \text { then } \quad y_{i}=-1
\end{array}\right.
$$

A separating hyperplane is defined by a normal vector $w$ and the offset $b$. The linear classifier is of the form,

$$
f(x)=w^{T} x+b
$$

Where, $w$ is called as the weight vector and the offset $b$ is the bias. Figure 2 shows the line of separation in 2dimentional space. It separates the space in to two classes, with the maximum margin.

\subsection{Test images}

The algorithm presented in the previous section is implemented in Matlab - R14 for identification and classification of the glaucoma disease. In our case of ocular thermal image, classification refers to the labelling of the given image into normal image or image with glaucoma. The ocular IR image data we used for SVM training in order to do the classification involves two categories: Set of Ocular image for left eye and set of ocular image for right eye. In our experiment, each set contained 15 normal images, and 15 patient images. All images are in JPEG format with size $128 \times$ 128. Figure 3 shows some samples from each image category: The input images from the database are first enhanced using Histogram equalization method and then the noise removal algorithm is used to remove the noise present.
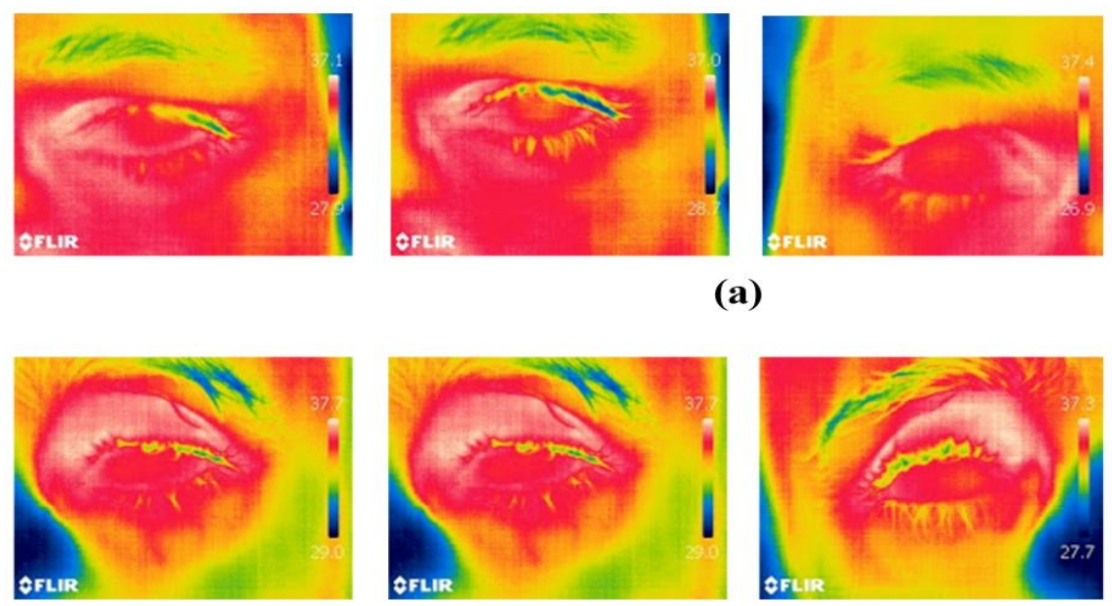

(a)
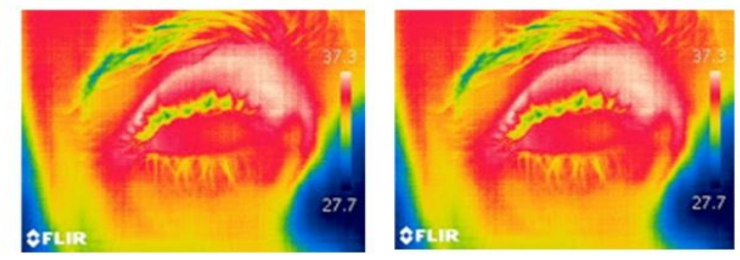

(b)

Fig. 3. Sample IR Thermal Eye Images used for training SVM

(a) Normal and (b) Patient (Glaucoma) eye images.
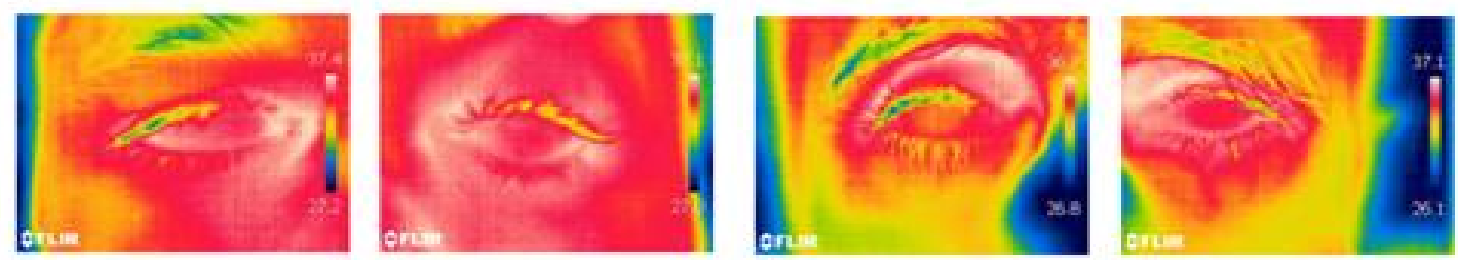

(a)

(b)

Fig. 4. Sample IR Thermal Eye Images used for testing (a) Normal and (b) Patient (Glaucoma) eye images. 


\subsection{Results and Discussion}

For the classification purpose, 2 sets of normal and patient ocular IR thermal images, each set having 15 images that are not part of training set are considered. Figure 4 shows some samples of the test image used. GLCM features extracted for few representative sample images are shown in table 1. We found that at almost at all instances the prediction performance of the test data set in to the classifier gave exact results and the classification accuracy rate is always the highest. This implies the feature vectors derived from GLCM matrix are more distinguishable for the two classes considered. It can be observed from table 1 that feature values related Energy and homogeneity are much more distinguishable compared to two other parameters namely contrast and correlation between the two sets. However, the classifier performance on the same dataset, with the two distinguishable parameters, the classifier performance is as good as when all the four parameters are considered.

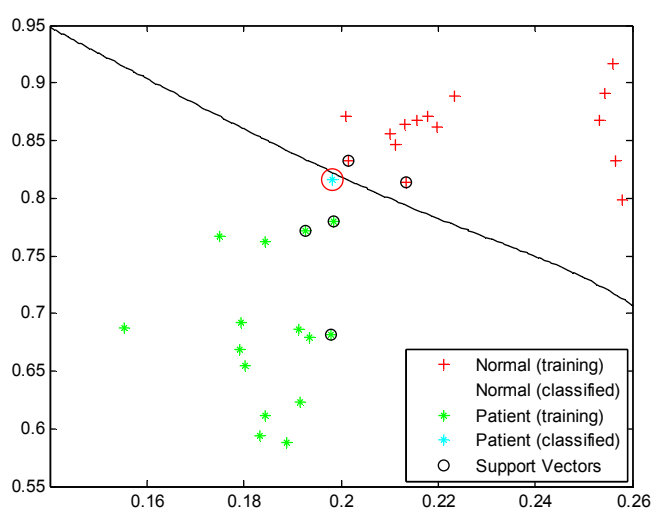

(a)

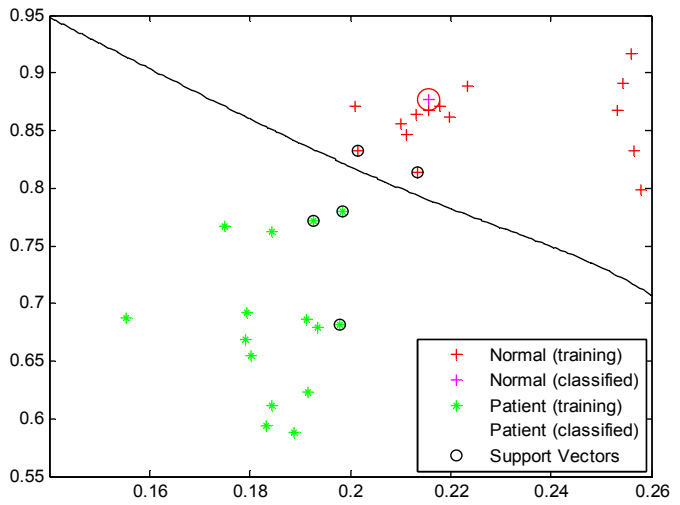

(b)

Fig. 5. Performance of the SVM Classifier (a) Classification of Normal ocular IR thermal image and

(b) Classification of Patient (Glaucoma) ocular IR thermal images.

Table 1. GLCM Feature vectors for few training IR images

\begin{tabular}{|l|l|l|l|l|}
\hline \multicolumn{5}{|c|}{ Normal Training Images } \\
\hline $\begin{array}{l}\text { Image } \\
\text { Features }\end{array}$ & Energy & Homogeneity & Contrast & Correlation \\
\hline Image 1 & 0.2011 & 0.8712 & 0.2144 & 0.8621 \\
\hline Image 2 & 0.2101 & 0.8561 & 0.2311 & 0.8645 \\
\hline Image 3 & 0.2113 & 0.8461 & 0.2287 & 0.8543 \\
\hline Image 4 & 0.2234 & 0.8891 & 0.2132 & 0.8671 \\
\hline Image 5 & 0.2132 & 0.8645 & 0.2287 & 0.8677 \\
\hline & & Patient Training Images & \\
\hline Image 1 & 0.1794 & 0.6921 & 0.2344 & 0.8631 \\
\hline Image 2 & 0.1801 & 0.6551 & 0.2211 & 0.8621 \\
\hline Image 3 & 0.1913 & 0.6861 & 0.2187 & 0.8423 \\
\hline Image 4 & 0.1934 & 0.6788 & 0.2282 & 0.8771 \\
\hline Image 5 & 0.1792 & 0.6685 & 0.2387 & 0.8897 \\
\hline
\end{tabular}

In Figure 5(a) and Figure 5(b), we show the graphical evidence for the performance of the SVM Classifier with the feature vector consisting of two most distinguishable feature parameters along with the SVM constructed an optimal hyperplane, in this case - a line as decision boundary. It can be observed that the support vector contains all the information needed to solve the classification task, even with a small database for training. 


\section{Conclusion}

This paper provides a methodology for the assessment of Glaucoma with Ocular Thermal images using GLCM and SVM based Classification. The algorithm uses features from GLCM which is used to train a simple linear SVM Classifier to evaluate a complex classification task for Glaucoma detection. Simulation results indicate the algorithm is efficient in terms of the classification accuracy rate, which is very high in identifying Glaucoma from normal eyes. The method is found to be simple and efficient. Further extensions in terms of increasing the number of parameters of GLCM and use of appropriate kernel for SVM may greatly increase the efficacy of the result obtained.

\section{Acknowledgements}

The authors like to thank IGCAR, India for the thermal images. Furthermore, the authors would like to thank the management of Sri Sivasubramania Nadar College of Engineering, kalavakkam, for providing the facilities to carry out this work.

\section{REFERENCES}

[1] Jen-Hong Tan., E.Y.K. Ng, Rajendra Acharya U, C. Chee, "Automated study of ocular thermal images: Comprehensive analysis of corneal health with different age group subjects and validation", Digital Signal Processing, pp.1579-1591, 2010.

[2] Jen Hong Tan, E. Y. K. Ng, U. Rajendra Acharya and Caroline Chee, "Infrared thermography on ocular Surface temperatures: A review", Infrared physics \& technology, vol-52, pp. 97-108, 2009.

[3] S.Kavitha, S.Karthikeyan and K.Duraiswamy, "Early Detection of Glaucoma in Retinal Images Using Cup to Disc Ratio", Second International conference on Computing, Communication and Networking Technologies, 2010.

[4] Tomi Kauppi, "Eye Fundus Image Analysis for automatic detection of Diabetic Retinopathy", Ph.D Thesis, 2010.

[5] Andrew James Patterson, "Analysis of Retinal Images in Glaucoma”,Ph.D Thesis, 2006.

[6] B. Wiecek, R. Danych, Z. Zwolenik, A. Jung, J. Zube,"Advanced Thermal Image Processing for medical and biological applications", Proceedings of the 23rd Annual EMBS International Conference, Istanbul, Turkey, 2001.

[7] Duarte, L. Carrão, M. Espanha, T. Viana, D. Freitas, P. Bártolo, P. Faria, and H.A. Almeida, "Segmentation algorithms for thermal images", Procedia Technology,vol.16, pp. 1560 - 1566, 2014.

[8] Philip B. Morgan, Meng Poey Soh, Nathan Efron and Andrew B. Tullos, "Potential Applications of ocular Thermography, Optometry and Vision Science", Vol.70, pp.568-576, 1993.

[9] Saheli Bhattacharya and N. Deepa, "Non-Contact Dry Eye Syndrome Diagnosis using Thermal Imaging and GLCM Feature", Proc. of Int. Conf. on Advances in Computer Science and Application, pp.759-763, 2013.

[10] Jen Hong Tan, E. Y. K. Ng, and U. Acharya, "An efficient automated algorithm to detect ocular surface temperature on sequence of thermograms using snake and target tracing function", Journal of medical systems, pp.949- 958, 2011.

[11] http://en.wikipedia.org/wiki/Support_vector_machine, 2015. 